\title{
The Physical Effects of Aromatherapy in Alleviating Work-Related Stress on Elementary School Teachers in Taiwan
}

\author{
Shing-Hong Liu, ${ }^{1}$ Tzu-Hsin Lin, ${ }^{2}$ and Kang-Ming Chang ${ }^{3,4}$ \\ ${ }^{1}$ Department of Computer Science and Information Engineering, Chaoyang University of Technology, Taichung, Taiwan \\ ${ }^{2}$ Department of Cardiology, Lin-Shin Hospital, Taichung, Taiwan \\ ${ }^{3}$ Department of Photonics and Communication Engineering, Asia University, Taichung, Taiwan \\ ${ }^{4}$ Graduate Institute of Clinical Medical Science, China Medical University, Taichung, Taiwan \\ Correspondence should be addressed to Tzu-Hsin Lin; linegg.linegg@msa.hinet.net and Kang-Ming Chang; changkm@asia.edu.tw
}

Received 24 July 2013; Revised 28 August 2013; Accepted 4 September 2013

Academic Editor: Gerhard Litscher

Copyright (c) 2013 Shing-Hong Liu et al. This is an open access article distributed under the Creative Commons Attribution License, which permits unrestricted use, distribution, and reproduction in any medium, provided the original work is properly cited.

People use aromatherapy to relieve the symptoms of physical and psychological stress. However, previous studies have not precisely clarified a scientific basis for the beneficial effects of aromatherapy. Therefore, the overall purpose of this study was to elucidate the beneficial effect of aromatherapy in relieving work-related stress. Twenty-nine elementary school teachers from Taiwan participated in this study. The experimental procedures comprised 2 phases. First, we verified the effect of aromatherapy by conducting 2 blind tests. We used natural bergamot essential oil extracted from plants and synthesized a chemical essential oil as the placebo to do the aromatherapy. Second, we analyzed the performance of the aromatherapy treatment on the teachers who had various workloads. We measured the teachers' heart rate variability to evaluate their autonomic nervous system activity. The results show that only the natural bergamot essential oil had an effect and that the aromatherapy treatment relieved work-related stress of teachers with various workloads. However, the aromatherapy treatment had a weak effect on young teachers who had a heavy workload. Moreover, the aromatherapy treatment exhibited no effect on teachers who belong to the abnormal body mass index subgroup having a heavy workload.

\section{Introduction}

In modern society, job-related stress is a substantial problem because $40 \%-50 \%$ of all relative work misses are related to stress [1]. Several previous studies have shown that the level of work-related stress could increase the risk of diseases such as cardiovascular disease $[2,3]$, neurodegenerative diseases [4], chronic diseases of aging [5], and metabolic syndrome [6]. Elementary school teachers in Taiwan must address problems related to Taiwan's low birth rate. In numerous Taiwanese families, there is only one child. Therefore, elementary school teachers must provide more time to process the child's problem except teaching. Moreover, teachers also do various administrative duties. Therefore, all elementary school teachers are at risk of suffering from work-related stress [7]. Thus, it is crucial for elementary schools to identify appropriate methods for coping with stress.
Various methods for coping with stress have been studied [8]. Aromatherapy is an appealing method because it is both effective and easy to implement [9]. Numerous previous studies have reported using essential oils to alleviate the symptoms of physical and psychological stress [10-13]. Among these studies, bergamot, lavender, and geranium were the most commonly applied essential oils, and the inhalation method was used more than other methods of delivery. These studies employed a randomized controlled trial to conduct the experiments and measured changes in the autonomic nervous systems of participants to quantify the performance of the aromatherapy treatment. During the experimental procedure, the participants were seated for 5 minutes after commencing the test, and their physiological data were collected. Subsequently, the aromatherapy group received the aromatherapy intervention for approximately 10-20 minutes, whereas the control group was either resting or inhaling 
from an empty diffuser. Following the test, the participants' data were collected. As discussed, the control group did not always use the placebo during the aromatherapy treatment for relieving physical stress symptoms. However, Lorig and Schwartz examined the relationship between the time-domain electroencephalograph (EEG) activity and selfreports from people exposed to various odorants [14]. The results indicate that the odors generated few perceptual or mood differences. EEG alpha and theta are the activity in the left and right hemispheres of the brain. Bagetta et al. used the brain wave spectrum power and found that bergamot essential oil correlates well with its exocytotic and carriermediated release of discrete amino acids endowed with the neurotransmitter function in the mammalian hippocampus [15]. Therefore, we considered the placebo a necessary factor for evaluating the effects of aromatherapy treatment.

The autonomic nervous system includes sympathetic and parasympathetic systems. When people feel physical or psychological stress, the sympathetic system becomes more active. When this stressor disappears, the parasympathetic system reduces the heart rate and the breathing rate. The response of the autonomic nervous system can be monitored using the heart rate variability (HRV), which is derived from heartbeat interval time series $[16,17]$. Some studies have shown that physical tasks influence the HRV $[1,18,19]$. Psychological depressive and anxiety disorders can also affect the HRV [20, 21]. Therefore, during aromatherapy treatment, the HRV is used to verify the effects of aromatherapy on physical and psychological tasks [10-13, 22].

Chang and Shen examined the effect of aromatherapy on elementary school teachers [10]. In their study, two conditions were not considered. (a) A placebo was not used in the experiment. They analyzed the HRV of subjects before and after aromatherapy treatment. If a synthetic essential oil is used in the treatment process, can it alleviate the symptoms of physical and psychological stress? (b) The varying workloads of subjects were not considered when conducting aromatherapy. Thus, quantifying the effect of aromatherapy is difficult.

The overall objective of our study was to clarify the beneficial effects of aromatherapy in alleviating work stress. The experiment comprised two phases. The purpose of the first phase was to verify the effect of aromatherapy by using two blind tests. We used natural essential oil extracted from plants and synthetic essential oil made with chemical materials to do the aromatherapy. The second phase involved analyzing the performance of aromatherapy for participants with varying workloads. To compare our results with those obtained by Chang and Shen, we also recruited elementary school teachers in Taiwan, and natural bergamot essential oil was used during treatment.

\section{Materials and Methods}

2.1. Participants. We recruited 29 elementary schoolteachers who did not have asthma, hypertension, or a heart condition. Because an aromatherapy spray may induce asthma, participants with a history of asthma were excluded. Moreover, numerous studies have shown that heart conditions such as arrhythmia, myocardial ischemia, and a history of heart failure affect the HRV [23-26]. Thus, participants with a heart disease and hypertension also were excluded from this study. The Beck Anxiety Inventory (BAI) was used to estimate the anxiety degree of every participant. The anxiety degree is considered minor when the score ranges between 0 and 7 . A score ranging between 8 and 15 indicates a light degree. A score ranging between 16 and 25 indicates a moderate degree, and a score between 26 and 63 indicates a serious degree.

2.2. Outcome Measures. For this study, we used $100 \%$ pure bergamot essential oil made in Italy, diluted to $2 \%$. The placebo was a synthetic essential oil (Shunyi Chemical Co., Ltd., Taiwan) with a similar scent to the bergamot essential oil. An ultrasonic ionizer aromatherapy diffuser was used for aroma evaporation (type YHL668/I, ultrasound frequency 2.5 MHz, Nature Creart Co., Ltd, Taiwan). For the heart rate measurements and HRV analysis, we employed a handheld HRV meter (LR8Z11, made by Yunyin Co., Ltd., Taiwan). It measures one lead electrocardiogram (ECG), the sampling rate is $500 \mathrm{~Hz}$, and the resolution is 12 bits. The blood pressure was measured using an electronic blood pressure monitor (type HEM-7210, made by OMRON Co., Ltd., Japan).

The HRV is derived from the heartbeat interval time series whose resampling is $4 \mathrm{~Hz}$ by using the further discrete Fourier transform. Low-frequency power (LF: 0.04-0.15 Hz), high-frequency power (HF: $0.15-0.4 \mathrm{~Hz}$ ), and the logarithmic ratio low- to high-frequency power $(\mathrm{LF} / \mathrm{HF})$ were calculated. We also used the normalized LF (LF\%) and HF (HF\%) to indicate the response of autonomic nervous activity. LF is affected by the vagal nervous and the sympathetic nervous, and $\mathrm{HF}$ is affected by the parasympathetic nervous [17].

2.3. Experiment Procedure. This experiment was approved by the Asia University Medical Research Ethics Committee. This was a two-phase experiment. The purpose of the first phase was to verify the effect of aromatherapy by conducting a blind test. The second experiment involved analyzing the effect of aromatherapy on teachers with varying workloads.

2.3.1. The Effect of Aromatherapy. In this experiment, each participant underwent the aroma treatment twice. Although the scent of the natural and synthetic essential oils is similar, certain people who carefully compare them at the same time can distinguish between them. Therefore, we set an interval of one week between the two experiments. To avoid the effect of different workloads, the aroma treatment for each participant was conducted on the same weekday at the same time. In both experiments, the participant did not know which essential oil was used. The experiment procedure is described below.

Step 1. Each participant was required to complete a consent form and a BAI survey and provide personal information including gender, age, years of employment, height, weight, and relationship status. Participants were required to abstain from smoking, alcohol, and coffee 6 hours before aroma treatment. At the resting time, the participants were then asked to sit in a chair and rest for approximately 3 minutes. 
Step 2. Their blood pressure was measured. We then measured the ECG lasting 3 minutes as a pretest. The participants kept their eyes open and remained still.

Step 3. The aroma treatment lasted approximately 15 minutes. The respiration rate and volume during this time were asked the same as the resting time.

Step 4. Blood pressure was again measured after completion of the aroma treatment. We then measured the ECG lasting 3 minutes as a posttest.

2.3.2. The Performance of Aromatherapy. In this experiment, we recruited the same participants. Each participant underwent aroma treatment twice: once during a heavy-workload state, and once more during a light-workload state. A heavy workload was defined as teaching more than four classes in one day, whereas a light workload was defined as teaching less than two classes in one day. All aroma treatment was conducted after the end of a day's classes. To allow independence for the two treatments, the second treatment was delayed one week from the first treatment. Natural bergamot essential oil was used. The experiment procedure is the same as in Section 2.3.1.

2.4. Statistics. We employed the SPSS 12.0 software package to conduct the one-way ANOVA analysis. Significance for the $P$ value was set at 0.05 . Descriptive statistics were represented as the mean \pm standard deviation. LF, HF, LF\%, HF\%, LF/HF, and the R-R interval time (RRI) were used to indicate the response of the autonomic nervous system. Zhang showed that age and gender affect the HRV [27]. Jarrett et al. indicated that the HRV is affected by the body mass index (BMI) [28]. Therefore, the intergroup differences among age, BMI, and the degree of anxiety were examined using an $F$ test.

\section{Results}

Detailed participant information is shown in Table 1. We examined 3 male participants and 26 female participants. The average age was $41.4 \pm 4$ years, and the average BMI was $22.2 \pm$ 3.6. The light-anxiety subgroup comprised 9 teachers, and the minor-anxiety subgroup contained 20 teachers. The older subgroup had 7 teachers, and the young subgroup had 18 teachers. Regarding BMI, the abnormal subgroup had 9 teachers, and the normal subgroup had 20 teachers.

The results obtained after natural bergamot essential oil treatment are shown in Table 2. The LF, LF\%, HF\%, and $\mathrm{LF} / \mathrm{HF}$ were found to have a significant difference. Table 3 shows that the results obtained for the synthetic essential oil did not have any indicators with a significant difference.

Tables 4 and 5 show the results before and after natural bergamot essential oil treatment for participants with light and heavy workloads, respectively. We can find that LF, LF\%, $\mathrm{HF} \%$, and LF/HF have significant differences. Therefore, the aromatherapy can alleviate the symptoms of physical and psychological stress at light and heavy workloads. Furthermore, after controlling the different variables, including age, BMI,
TABLE 1: Participant information $(n=29)$.

\begin{tabular}{lc}
\hline Items & People number \\
\hline \multirow{2}{*}{ Gender } & Male: 3 \\
& Female: 26 \\
BAI & Minor: 20 \\
& Light: 9 \\
Age (years) & Elder $(>40): 18$ \\
& Young $(\leq 40): 11$ \\
BMI $\left(\mathrm{Kg} / \mathrm{m}^{2}\right)$ & $>24: 9$ \\
& $\leq 24$ and $\geq 21: 20$ \\
\hline
\end{tabular}

and anxiety degree, we conducted an analysis of the subgroup regarding the performance of aromatherapy, the results of which are described as follows. Table 6 shows the results of the age subgroups with a light workload. LF, LF\%, HF\%, and $\mathrm{LF} / \mathrm{HF}$ all show a significant difference for the young and older subgroups. Table 7 shows the results for heavy workload teachers. Only the older subgroup was shown to be affected by aromatherapy, with LF, LF\%, HF\%, and LF/HF having a significant difference. However, in the young subgroup, none of the indicators, except for LF, were found to have a significant difference. Table 8 shows the results of the BMI subgroups with a light workload. The indicators, LF, $\mathrm{LF} \%, \mathrm{HF} \%$, and LF/HF, have a significant difference in the normal and abnormal BMI subgroups. Table 9 shows the results for teachers with a heavy workload. Only the normal BMI subgroup was shown to be affected by aromatherapy; $\mathrm{LF}, \mathrm{LF} \%, \mathrm{HF} \%$, and LF/HF have a significant difference in this subgroup. In the abnormal BMI subgroup, none of the indicators showed a significant difference. Tables 10 and 11 show the analyzed results of the anxiety-degree subgroups with light and heavy workloads. LF, LF\%, HF\%, and LF/HF were all found to have a significant difference.

\section{Discussions}

Chang and Shen examined the effect of aromatherapy on elementary schoolteachers in Taiwan [10]. Although their results were positive, certain research points were not considered clearly, such as placebo use and teachers' varying workloads. The focus of our study was on the physical effect of aromatherapy on elementary schoolteachers with different workloads. We added a placebo variable to determine whether the HRV response was actually obtained from the aromatherapy. The placebo was a synthetic essential oil that has a similar scent to natural bergamot essential oil. We then examined the physical effect of aromatherapy on alleviating the work stress of teachers with different workloads. Because the age, BMI, and anxiety degree could affect the HRV, we also analyzed these subgroups. Chang and Shen used the ANS Watch monitor to measure the HRV and blood pressure. This device measures the pulse wave of the radial artery to detect the HRV. In contrast, we used an ECG to detect the HRV. Thus, the data of certain indicators differ.

In this study, the HF indicator did not show a significant difference in the two experiments. Because the respiration 
TABLE 2: The HRV one-way ANOVA results before and after natural bergamot essential oil treatment for all participants.

\begin{tabular}{lcccc}
\hline Indicators & Before $(n=29)$ & After $(n=29)$ & $F$ & $P$ \\
\hline LF $\left(\mathrm{ms}^{2}\right)$ & $9.80 \pm 1.19$ & $9.05 \pm 1.31$ & 15.21 & $0.00^{* *}$ \\
HF $\left(\mathrm{ms}^{2}\right)$ & $8.91 \pm 1.45$ & $8.96 \pm 1.36$ & 0.04 & 0.840 \\
LF/HF (nu) & $0.88 \pm 1.00$ & $0.10 \pm 1.10$ & 24.34 & $0.00^{* *}$ \\
LF\% (nu) & $68.6 \pm 19.4$ & $52.2 \pm 22.2$ & 26.76 & $0.00^{* *}$ \\
HF\% (nu) & $32.3 \pm 19.5$ & $48.7 \pm 22.1$ & 26.92 & $0.00^{* *}$ \\
RRI (ms) & $766.3 \pm 90.2$ & $781.5 \pm 98.4$ & 1.12 & 0.290 \\
\hline
\end{tabular}

${ }^{*} P<0.05,{ }^{* *} P<0.01$.

TABLE 3: The HRV one-way ANOVA results before and after synthetic essential oil treatment for all participants.

\begin{tabular}{lccrr}
\hline Indicators & Before $(n=29)$ & After $(n=29)$ & $F$ & $P$ \\
\hline LF $\left(\mathrm{ms}^{2}\right)$ & $9.5 \pm 1.1$ & $9.3 \pm 1.2$ & 0.43 & 0.99 \\
HF $\left(\mathrm{ms}^{2}\right)$ & $8.8 \pm 1.2$ & $9.0 \pm 1.2$ & 3.27 & 0.311 \\
LF/HF (nu) & $0.64 \pm 1.01$ & $0.34 \pm 1.13$ & 3.54 & 0.075 \\
LF\% (nu) & $63.25 \pm 20.11$ & $57.09 \pm 22.94$ & 3.54 & 0.061 \\
HF\% (nu) & $37.45 \pm 20.10$ & $43.64 \pm 23.05$ & 2.48 & 0.061 \\
RRI (ms) & $740.1 \pm 85.9$ & $760.2 \pm 85.7$ & 0.124 \\
\hline
\end{tabular}

${ }^{*} P<0.05,{ }^{* *} P<0.01$.

TABLE 4: The HRV one-way ANOVA results before and after natural bergamot essential oil treatment for all participants with a light workload.

\begin{tabular}{lcccc}
\hline Indicators & Before $(n=29)$ & After $(n=29)$ & $F$ & $P$ \\
\hline LF $\left(\mathrm{ms}^{2}\right)$ & $9.74 \pm 1.18$ & $8.97 \pm 1.30$ & 16.96 & $0.000^{* *}$ \\
HF $\left(\mathrm{ms}^{2}\right)$ & $8.98 \pm 1.49$ & $8.92 \pm 1.39$ & 20.13 & 0.790 \\
LF/HF (nu) & $0.76 \pm 0.97$ & $0.04 \pm 1.14$ & 21.69 & $0.000^{* *}$ \\
LF\% (nu) & $65.85 \pm 20.35$ & $50.68 \pm 22.57$ & 24.21 & $0.000^{* *}$ \\
HF\% (nu) & $34.45 \pm 19.88$ & $50.27 \pm 22.43$ & 0.09 & $0.000^{* *}$ \\
RRI (ms) & $773 \pm 96.4$ & $778 \pm 102$ & & 0.763 \\
\hline
\end{tabular}

${ }^{*} P<0.05,{ }^{* *} P<0.01$.

TABLE 5: The HRV one-way ANOVA results before and after natural bergamot essential oil treatment for all participants with a heavy workload.

\begin{tabular}{lcccc}
\hline Indicators & Before $(n=29)$ & After $(n=29)$ & $F$ & \multicolumn{1}{c}{} \\
\hline LF $\left(\mathrm{ms}^{2}\right)$ & $9.68 \pm 1.15$ & $9.12 \pm 1.13$ & 10.41 & $0.001^{* *}$ \\
HF $\left(\mathrm{ms}^{2}\right)$ & $8.98 \pm 1.59$ & $9.26 \pm 1.27$ & 1.68 & 0.196 \\
LF/HF $(\mathrm{nu})$ & $0.70 \pm 1.15$ & $-0.14 \pm 1.16$ & 23.04 & $0.000^{* *}$ \\
LF\% $(\mathrm{nu})$ & $63.6 \pm 22.3$ & $47.3 \pm 24.0$ & 21.47 & $0.000^{* *}$ \\
HF\% (nu) & $37.2 \pm 22.4$ & $53.4 \pm 23.9$ & 21.42 & $0.000^{* *}$ \\
RRI (ms) & $771 \pm 114$ & $7821 \pm 109$ & 0.36 & 0.548 \\
\hline
\end{tabular}

${ }^{*} P<0.05,{ }^{* *} P<0.01$.

TABLE 6: The HRV one-way ANOVA results of the age subgroups with a light workload before and after natural bergamot essential oil treatment for all participants.

\begin{tabular}{|c|c|c|c|c|c|c|c|c|}
\hline \multirow{2}{*}{ Indicators } & \multicolumn{4}{|c|}{ Elder $(n=18)$} & \multicolumn{4}{|c|}{ Young $(n=11)$} \\
\hline & Before & After & $F$ & $P$ & Before & After & $F$ & $P$ \\
\hline $\mathrm{LF}\left(\mathrm{ms}^{2}\right)$ & $9.74 \pm 1.15$ & $9.06 \pm 1.41$ & 7.50 & $0.007^{* *}$ & $9.75 \pm 1.25$ & $8.81 \pm 1.10$ & 10.42 & $0.002^{* *}$ \\
\hline $\mathrm{HF}\left(\mathrm{ms}^{2}\right)$ & $8.70 \pm 1.42$ & $8.70 \pm 1.38$ & 0.00 & 0.995 & $9.45 \pm 1.52$ & $9.29 \pm 1.36$ & 0.19 & 0.660 \\
\hline LF/HF (nu) & $1.04 \pm 0.82$ & $0.36 \pm 1.13$ & 12.71 & $0.001^{* *}$ & $0.30 \pm 1.02$ & $-0.48 \pm 0.94$ & 10.41 & $0.002^{* *}$ \\
\hline LF\% (nu) & $71.2 \pm 17.5$ & $57.1 \pm 21.8$ & 13.59 & $0.000^{* *}$ & $57.2 \pm 21.9$ & $40.2 \pm 19.9$ & 10.92 & $0.002^{* *}$ \\
\hline HF\% (nu) & $28.7 \pm 16.5$ & $43.1 \pm 21.8$ & 16.61 & $0.000^{* *}$ & $43.8 \pm 21.6$ & $60.7 \pm 19.6$ & 11.04 & $0.001^{* *}$ \\
\hline RRI (ms) & $763 \pm 74.7$ & $771 \pm 76.5$ & 0.36 & 0.552 & $792 \pm 123$ & $790 \pm 136$ & 0.01 & 0.946 \\
\hline
\end{tabular}

${ }^{*} P<0.05,{ }^{* *} P<0.01$. 
TABLE 7: The HRV one-way ANOVA results of the age subgroups with a heavy workload before and after natural bergamot essential oil treatment for all participants.

\begin{tabular}{|c|c|c|c|c|c|c|c|c|}
\hline \multirow{2}{*}{ Indicators } & \multicolumn{4}{|c|}{ Elder $(n=18)$} & \multicolumn{4}{|c|}{ Young $(n=11)$} \\
\hline & Before & After & $F$ & P & Before & After & $F$ & $P$ \\
\hline $\mathrm{LF}\left(\mathrm{ms}^{2}\right)$ & $9.55 \pm 1.23$ & $9.08 \pm 1.16$ & 4.258 & $0.042^{*}$ & $9.89 \pm 0.98$ & $9.19 \pm 1.10$ & 7.32 & $0.009^{* *}$ \\
\hline $\mathrm{HF}\left(\mathrm{ms}^{2}\right)$ & $8.55 \pm 1.67$ & $9.11 \pm 1.30$ & 3.71 & 0.057 & $9.67 \pm 1.17$ & $9.51 \pm 1.20$ & 0.31 & 0.583 \\
\hline LF/HF (nu) & $1.00 \pm 1.18$ & $-0.03 \pm 1.07$ & 22.53 & $0.000^{* *}$ & $0.21 \pm 0.91$ & $-0.32 \pm 1.30$ & 3.71 & 0.058 \\
\hline LF\% (nu) & $69.0 \pm 22.2$ & $49.6 \pm 22.9$ & 20.02 & $0.000^{* *}$ & $54.8 \pm 19.8$ & $43.6 \pm 25.7$ & 3.91 & 0.052 \\
\hline HF\% (nu) & $31.7 \pm 22.4$ & $51.2 \pm 22.9$ & 20.15 & $0.000^{* *}$ & $46.1 \pm 19.7$ & $57.0 \pm 25.4$ & 3.78 & 0.056 \\
\hline RRI (ms) & $759 \pm 86.2$ & $769 \pm 76.4$ & 0.46 & 0.498 & $792 \pm 148$ & $801 \pm 147$ & 0.07 & 0.797 \\
\hline
\end{tabular}

${ }^{*} P<0.05,{ }^{* *} P<0.01$.

TABLE 8: The HRV one-way ANOVA results of the BMI subgroups with a light workload before and after natural bergamot essential oil treatment for all participants.

\begin{tabular}{|c|c|c|c|c|c|c|c|c|}
\hline \multirow{2}{*}{ Indicators } & \multicolumn{4}{|c|}{$\mathrm{BMI}>40(n=9)$} & \multicolumn{4}{|c|}{$21 \leq \mathrm{BMI} \leq 24(n=20)$} \\
\hline & Before & After & $F$ & $P$ & Before & After & $F$ & $P$ \\
\hline $\mathrm{LF}\left(\mathrm{ms}^{2}\right)$ & $9.7 \pm 1.6$ & $8.8 \pm 1.6$ & 4.59 & $0.037^{*}$ & $9.8 \pm 0.9$ & $9.1 \pm 1.2$ & 13.46 & $0.000^{* *}$ \\
\hline $\mathrm{HF}\left(\mathrm{ms}^{2}\right)$ & $8.71 \pm 2.09$ & $8.63 \pm 2.05$ & 0.02 & 0.895 & $9.11 \pm 1.13$ & $9.1 \pm 1.0$ & 0.07 & 0.790 \\
\hline $\mathrm{LF} / \mathrm{HF}$ (nu) & $0.97 \pm 0.77$ & $0.12 \pm 0.97$ & 12.89 & $0.001^{* *}$ & $0.66 \pm 1.04$ & $0.01 \pm 1.21$ & 10.16 & $0.002^{* *}$ \\
\hline LF\% (nu) & $71.1 \pm 15.4$ & $52.3 \pm 20.1$ & 14.95 & $0.000^{* *}$ & $63.5 \pm 21.94$ & $50.0 \pm 23.7$ & 10.53 & $0.002^{* *}$ \\
\hline $\mathrm{HF} \%$ (nu) & $29.9 \pm 15.5$ & $48.7 \pm 20.4$ & 14.47 & $0.000^{* *}$ & $36.5 \pm 21.36$ & $51.0 \pm 23.4$ & 12.53 & $0.001^{* *}$ \\
\hline RRI (ms) & $767 \pm 139$ & $773 \pm 163$ & 0.02 & 0.888 & $777 \pm 70.1$ & $781 \pm 60.2$ & 0.11 & 0.740 \\
\hline
\end{tabular}

${ }^{*} P<0.05,{ }^{* *} P<0.01$.

TABLE 9: The HRV one-way ANOVA results of the BMI subgroups with a heavy workload before and after natural bergamot essential oil treatment for all participants.

\begin{tabular}{lcccccccc}
\hline \multirow{2}{*}{ Indicators } & \multicolumn{3}{c}{ BMI $>40(n=9)$} & \multicolumn{3}{c}{$21 \leq \mathrm{BMI} \leq 24(n=20)$} \\
& Before & After & $F$ & $P$ & Before & After & $F$ \\
\hline LF $\left(\mathrm{ms}^{2}\right)$ & $9.40 \pm 1.30$ & $9.33 \pm 1.13$ & 0.05 & 0.817 & $9.80 \pm 1.06$ & $9.02 \pm 1.13$ & 14.93 & $0.000^{* *}$ \\
HF $\left(\mathrm{ms}^{2}\right)$ & $8.88 \pm 1.93$ & $8.96 \pm 1.48$ & 0.08 & 0.774 & $9.04 \pm 1.43$ & $9.40 \pm 1.16$ & 2.18 & 0.143 \\
LF/HF (nu) & $0.58 \pm 0.93$ & $0.37 \pm 0.91$ & 0.71 & 0.402 & $0.76 \pm 1.24$ & $-0.37 \pm 1.20$ & 25.60 & $0.000^{* *}$ \\
LF\% (nu) & $62.4 \pm 19.1$ & $58.6 \pm 20.1$ & 0.50 & 0.481 & $64.2 \pm 23.7$ & $42.3 \pm 24.1$ & 25.20 & $0.000^{* *}$ \\
HF\% (nu) & $38.7 \pm 19.6$ & $42.4 \pm 20.3$ & 0.45 & 0.507 & $36.4 \pm 23.7$ & $58.4 \pm 23.8$ & 25.54 & $0.000^{* *}$ \\
RRI (ms) & $768 \pm 153$ & $802 \pm 152$ & 0.64 & 0.426 & $773 \pm 93.0$ & $772 \pm 82.9$ & 0.00 & 0.989 \\
\hline
\end{tabular}

${ }^{*} P<0.05,{ }^{* *} P<0.01$.

frequency at a resting time is within $0.1 \sim 0.4 \mathrm{~Hz}$, which locates at the range of $\mathrm{HF}$, the calculated power of $\mathrm{HF}$ will include the power of respiratory frequency [29]. Therefore, some studies of aromatherapy have used only the $\mathrm{LH}$ and LF/HF indicators to identify the effect of aromatherapy $[10,13]$. In this study, we focused on the LF, LF/HF, LF\%, and $\mathrm{HF} \%$ indicators to analyze the effect and performance of aromatherapy.

According to Tables 2 and 3, although the participants could not distinguish between the scent of the natural essential oil and synthetic essential oil, the natural bergamot essential oil has a relaxing effect, whereas the synthetic essential oil does not. This result directly shows that the essential bergamot oil extracted from natural materials for aromatherapy could be a better choice.

Tables 4 and 5 show the effect of aromatherapy on reducing work stress according to varying workloads. The results are in agreement with those of Chang and Shen [10]. In the age subgroup, the light-workload results are in agreement with those of Chang and Shen [10]. However, the elder subgroup with a heavy workload had a better performance than the young subgroup by the aromatherapy. This result differs from the findings of Chang and Shen because Chang and Shen did not consider differences in subjects' workloads; their results for all age subgroups showed that aromatherapy caused an effect [10]. Zhang demonstrated that age had a greater impact on HRV than gender. The older age group had a consistently lower LF and HF compared with the younger group [27]. In our study, the LF\% and LF/HF of the young subgroup decreased, and $\mathrm{HF} \%$ increased before and after aroma treatment. However, none of these indicators were found to have a significant difference. Therefore, age variable may affect the performance of aromatherapy for teachers with a heavy workload. In the BMI analysis, the aromatherapy had effect on teachers with a light workload. But the HRV did not have the significant change before and after aroma treatment with a heavy workload. This is a very interesting 
TABLE 10: The HRV one-way ANOVA results of the anxiety-degree subgroups with a light workload before and after natural bergamot essential oil treatment for all participants.

\begin{tabular}{|c|c|c|c|c|c|c|c|c|}
\hline \multirow{2}{*}{ Indicators } & \multicolumn{4}{|c|}{ Minor $(n=20)$} & \multicolumn{4}{|c|}{ Light $(n=9)$} \\
\hline & Before & After & $F$ & $P$ & Before & After & $F$ & $P$ \\
\hline $\mathrm{LF}\left(\mathrm{ms}^{2}\right)$ & $9.72 \pm 1.24$ & $9.12 \pm 1.36$ & 6.27 & $0.014^{*}$ & $9.80 \pm 1.08$ & $8.62 \pm 1.10$ & 15.85 & $0.000^{* *}$ \\
\hline $\mathrm{HF}\left(\mathrm{ms}^{2}\right)$ & $8.95 \pm 1.54$ & $8.89 \pm 1.43$ & 0.04 & 0.834 & $9.06 \pm 1.40$ & $9.00 \pm 1.33$ & 0.03 & 0.870 \\
\hline LF/HF (nu) & $0.77 \pm 1.03$ & $0.23 \pm 1.24$ & 6.69 & $0.011^{*}$ & $0.74 \pm 0.82$ & $-0.38 \pm 0.72$ & 28.01 & $0.000^{* *}$ \\
\hline LF\% (nu) & $65.6 \pm 21.7$ & $54.4 \pm 24.4$ & 6.94 & $0.010^{*}$ & $66.5 \pm 17.4$ & $42.3 \pm 15.0$ & 29.99 & $0.000^{* *}$ \\
\hline HF\% (nu) & $34.3 \pm 21.0$ & $46.6 \pm 24.3$ & 8.73 & $0.004^{* *}$ & $34.8 \pm 17.4$ & $58.5 \pm 14.8$ & 29.02 & $0.000^{* *}$ \\
\hline RRI (ms) & $778 \pm 102$ & $788 \pm 115$ & 0.24 & 0.626 & $763 \pm 82.5$ & $756 \pm 64.5$ & 0.12 & 0.733 \\
\hline
\end{tabular}

${ }^{*} P<0.05,{ }^{* *} P<0.01$.

TABLE 11: The HRV one-way ANOVA results of the anxiety-degree subgroups with a heavy workload before and after natural bergamot essential oil treatment for all participants.

\begin{tabular}{|c|c|c|c|c|c|c|c|c|}
\hline \multirow{2}{*}{ Indicators } & \multicolumn{4}{|c|}{$\operatorname{Minor}(n=20)$} & \multicolumn{4}{|c|}{ Light $(n=9)$} \\
\hline & Before & After & $F$ & $P$ & Before & After & $F$ & $P$ \\
\hline $\mathrm{LF}\left(\mathrm{ms}^{2}\right)$ & $9.62 \pm 1.27$ & $9.14 \pm 1.23$ & 4.28 & $0.041^{*}$ & $9.82 \pm 0.81$ & $9.07 \pm 0.88$ & 10.46 & $0.002^{* *}$ \\
\hline $\mathrm{HF}\left(\mathrm{ms}^{2}\right)$ & $8.83 \pm 1.66$ & $9.21 \pm 1.33$ & 1.91 & 0.170 & $9.31 \pm 1.40$ & $9.38 \pm 1.15$ & 0.04 & 0.841 \\
\hline LF/HF (nu) & $0.79 \pm 1.18$ & $-0.06 \pm 1.25$ & 14.82 & $0.000^{* *}$ & $0.51 \pm 1.08$ & $-0.31 \pm 0.95$ & 8.74 & $0.005^{* *}$ \\
\hline LF\% (nu) & $65.3 \pm 22.9$ & $49.2 \pm 25.3$ & 13.32 & $0.000^{* *}$ & $60.0 \pm 20.8$ & $43.2 \pm 20.8$ & 8.75 & $0.005^{* *}$ \\
\hline $\mathrm{HF} \%$ (nu) & $35.4 \pm 23.0$ & $51.6 \pm 25.2$ & 13.59 & $0.000^{* *}$ & $41.1 \pm 21.1$ & $57.5 \pm 20.7$ & 8.30 & $0.006^{* *}$ \\
\hline RRI (ms) & $77.3 \pm 9.5$ & $74.7 \pm 11.6$ & 0.08 & 0.783 & $68.3 \pm 9.6$ & $66.9 \pm 9.6$ & 0.94 & 0.337 \\
\hline
\end{tabular}

${ }^{*} P<0.05,{ }^{* *} P<0.01$.

result. Jarrett et al. indicated that age and BMI affect the HRV [28]. However, this was not within the scope of our study.

\section{Conclusion}

We conducted this trial to examine the potential of using natural bergamot essential oil in appeasing the work stress of elementary schoolteachers. The response of automatic nervous system has a significant change after the natural essential bergamot oil treatment. We also analyzed the effect of aromatherapy at different workloads. The aromatherapy may alleviate the symptoms of physical and psychological stress. The results also suggest that age and BMI factors affect aromatherapy performance when teachers have a heavy workload.

\section{Acknowledgment}

This work has been supported by the National Science Council of Taiwan (Grants nos. NSC 102-2221-E-324-004and NSC 102-2221-E-468-003-).

\section{References}

[1] J. Taelman, S. Vandeput, E. Vlemincx, A. Spaepen, and S. van Huffel, "Instantaneous changes in heart rate regulation due to mental load in simulated office work," European Journal of Applied Physiology, vol. 111, no. 7, pp. 1497-1505, 2011.

[2] M. Kivimäki, "Work stress and risk of cardiovascular mortality: prospective cohort study of industrial employees," British Medical Journal, vol. 325, no. 7369, pp. 857-860, 2002.
[3] T. Esch, G. B. Stefano, G. L. Fricchione, and H. Benson, "Stress in cardiovascular diseases," Medical Science Monitor, vol. 8, no. 5, pp. RA93-RA101, 2002.

[4] T. Esch, G. B. Stefano, G. L. Fricchione, and H. Benson, "The role of stress in neurodegenerative diseases and mental disorders," Neuroendocrinology Letters, vol. 23, no. 3, pp. 199-208, 2002.

[5] G. E. Miller, E. Chen, and K. J. Parker, "Psychological stress in childhood and susceptibility to the chronic diseases of aging: moving toward a model of behavioral and biological mechanisms," Psychological Bulletin, vol. 137, no. 6, pp. 959-997, 2011.

[6] R. Rosmond, "Role of stress in the pathogenesis of the metabolic syndrome," Psychoneuroendocrinology, vol. 30, no. 1, pp. 1-10, 2005.

[7] Y. H. Kuo, The study of the relationships between the job stresses and burnout in the primary school teachers [M.S. thesis], National Pingtung University of Education, Pingtung, Taiwan, 2004.

[8] K. Shader, M. E. Broome, C. D. Broome, M. E. West, and M. Nash, "Factors influencing satisfaction and anticipated turnover for nurses in an academic medical center," Journal of Nursing Administration, vol. 31, no. 4, pp. 210-216, 2001.

[9] B. Viktor and S. Gretchen, Aromatherapy, Avon Books, New York, NY, USA, 1993.

[10] K. Chang and C. Shen, "Aromatherapy benefits autonomic nervous system regulation for elementary school faculty in Taiwan," Evidence-Based Complementary and Alternative Medicine, vol. 2011, Article ID 946537, 7 pages, 2011.

[11] V. W. C. Yim, A. K. Y. Ng, H. W. H. Tsang, and A. Y. Leung, "A review on the effects of aromatherapy for patients with depressive symptoms," The Journal of Alternative and Complementary Medicine, vol. 15, no. 2, pp. 187-195, 2009. 
[12] Y. Lee, Y. Wu, H. W. H. Tsang, and A. Y. Leung, "A systematic review on the anxiolytic effects of aromatherapy in people with anxiety symptoms," The Journal of Alternative and Complementary Medicine, vol. 17, no. 2, pp. 101-108, 2011.

[13] T. Igarashi, "Physical and psychologic effects of aromatherapy inhalation on pregnant women: a randomized controlled trial," The Journal of Alternative and Complementary Medicine, 2013.

[14] T. S. Lorig and G. E. Schwartz, "Brain and odor: I. Alteration of human EEG by odor administration," Psychobiology, vol. 16, no. 3, pp. 281-284, 1988.

[15] G. Bagetta, L. A. Morrone, L. Rombolà et al., "Neuropharmacology of the essential oil of bergamot," Fitoterapia, vol. 81, no. 6, pp. 453-461, 2010.

[16] S. Akselrod, D. Gordon, F. A. Ubel, D. C. Shannon, A. C. Barger, and R. J. Cohen, "Power spectrum analysis of heart rate fluctuation: a quantitative probe of beat-to-beat cardiovascular control," Science, vol. 213, no. 4504, pp. 220-222, 1981.

[17] Task Force of the European Society of Cardiology and the North American Society of Pacing and Electrophysiology, "Heart rate variability: standards of measurement, physiological interpretation, and clinical use," Circulation, vol. 93, no. 5, pp. 1043-1065, 1996.

[18] R. Perini, S. Milesi, N. M. Fisher, D. R. Pendergast, and A. Veicsteinas, "Heart rate variability during dynamic exercise in elderly males and females," European Journal of Applied Physiology, vol. 82, no. 1-2, pp. 8-15, 2000.

[19] M. N. Bartels, S. Jelic, P. Ngai et al., "The effect of ventilation on spectral analysis of heart rate and blood pressure variability during exercise," Respiratory Physiology and Neurobiology, vol. 144, no. 1, pp. 91-98, 2004.

[20] J. M. Gorman and R. P. Sloan, "Heart rate variability in depressive and anxiety disorders," American Heart Journal, vol. 140, pp. S77-S83, 2000.

[21] K. Narita, T. Murata, T. Hamada et al., "Interactions among higher trait anxiety, sympathetic activity, and endothelial function in the elderly," Journal of Psychiatric Research, vol. 41, no. 5, pp. 418-427, 2007.

[22] H. Takeda, J. Tsujita, M. Kaya, M. Takemura, and Y. Oku, "Differences between the physiologic and psychologic effects of aromatherapy body treatment," The Journal of Alternative and Complementary Medicine, vol. 14, no. 6, pp. 655-661, 2008.

[23] M. M. Wolf, G. A. Varigos, D. Hunt, and J. G. Sloman, "Sinus arrhythmia in acute myocardial infarction," Medical Journal of Australia, vol. 2, no. 2, pp. 52-53, 1978.

[24] F. Lombardi, G. Sandrone, M. T. Spinnler et al., "Heart rate variability in the early hours of an acute myocardial infarction," American Journal of Cardiology, vol. 77, no. 12, pp. 1037-1044, 1996.

[25] D. Lucini, G. S. Mela, A. Malliani, and M. Pagani, "Impairment in cardiac autonomic regulation preceding arterial hypertension in humans: insights from spectral analysis of beat-by-beat cardiovascular variability," Circulation, vol.106, no. 21, pp. 26732679, 2002.

[26] D. Lucini, R. V. Milani, G. Costantino, C. J. Lavie, A. Porta, and M. Pagani, "Effects of cardiac rehabilitation and exercise training on autonomic regulation in patients with coronary artery disease," American Heart Journal, vol. 143, no. 6, pp. 977983, 2002.

[27] J. Zhang, "Effect of age and sex on heart rate variability in healthy subjects," Journal of Manipulative and Physiological Therapeutics, vol. 30, no. 5, pp. 374-379, 2007.
[28] M. E. Jarrett, R. L. Burr, K. C. Cain, J. D. Rothermel, C. A. Landis, and M. M. Heitkemper, "Autonomic nervous system function during sleep among women with irritable bowel syndrome," Digestive Diseases and Sciences, vol. 53, no. 3, pp. 694-703, 2007.

[29] N. Montano, A. Porta, C. Cogliati et al., "Heart rate variability explored in the frequency domain: a tool to investigate the link between heart and behavior," Neuroscience and Biobehavioral Reviews, vol. 33, no. 2, pp. 71-80, 2009. 


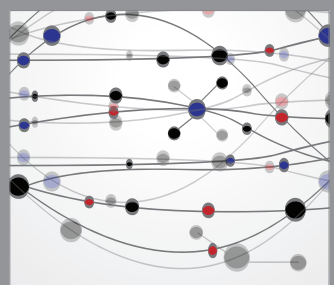

The Scientific World Journal
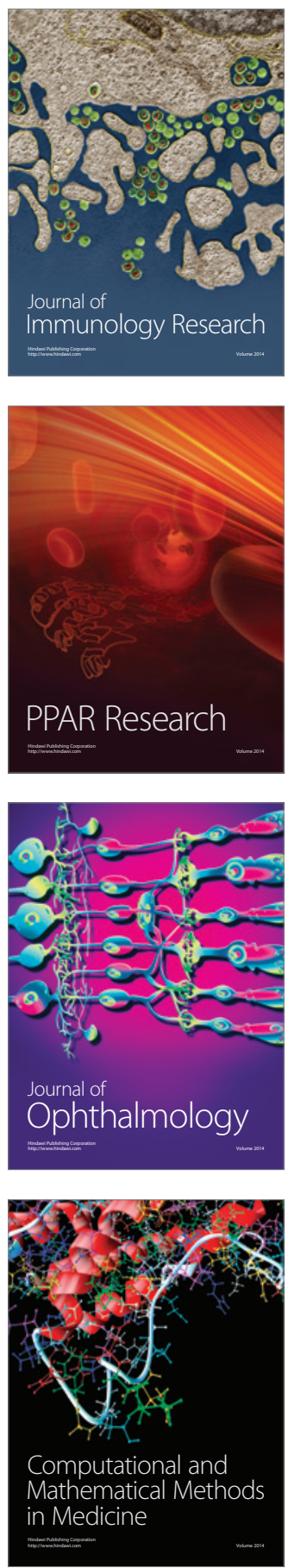

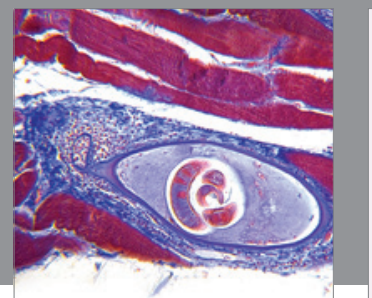

Gastroenterology

Research and Practice
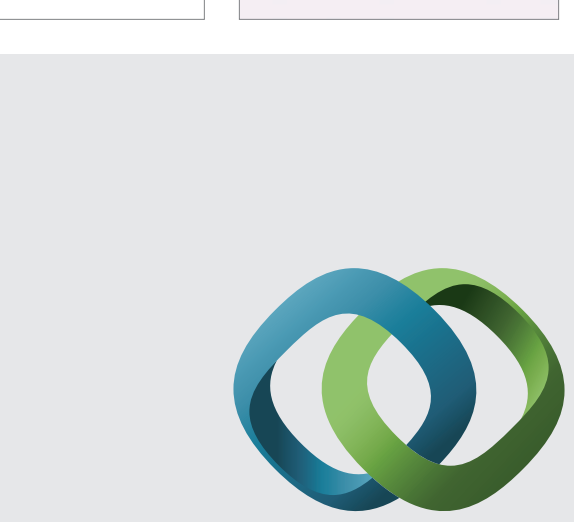

\section{Hindawi}

Submit your manuscripts at

http://www.hindawi.com
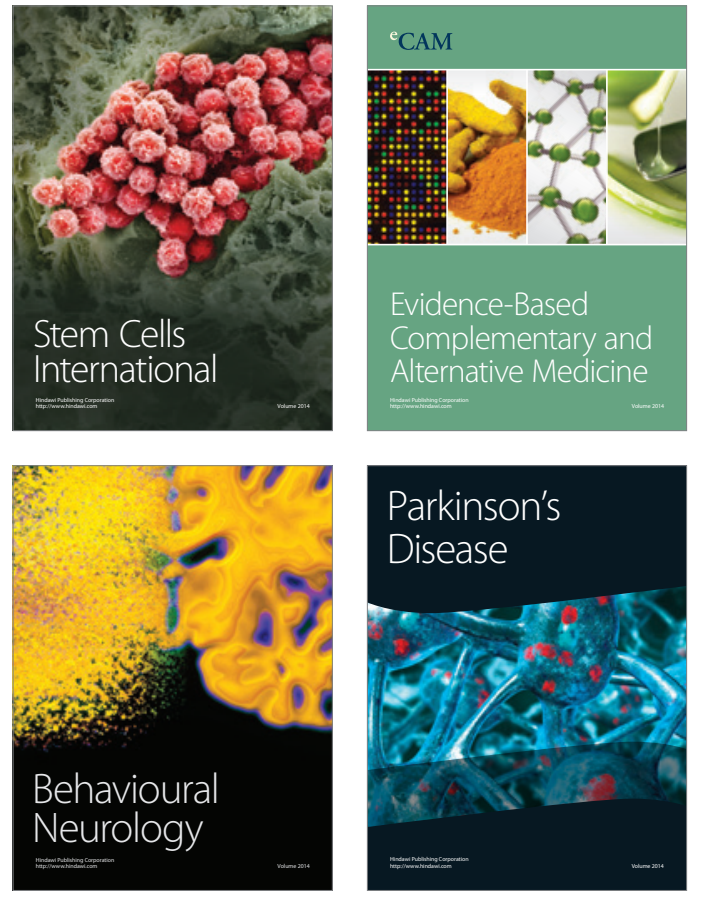
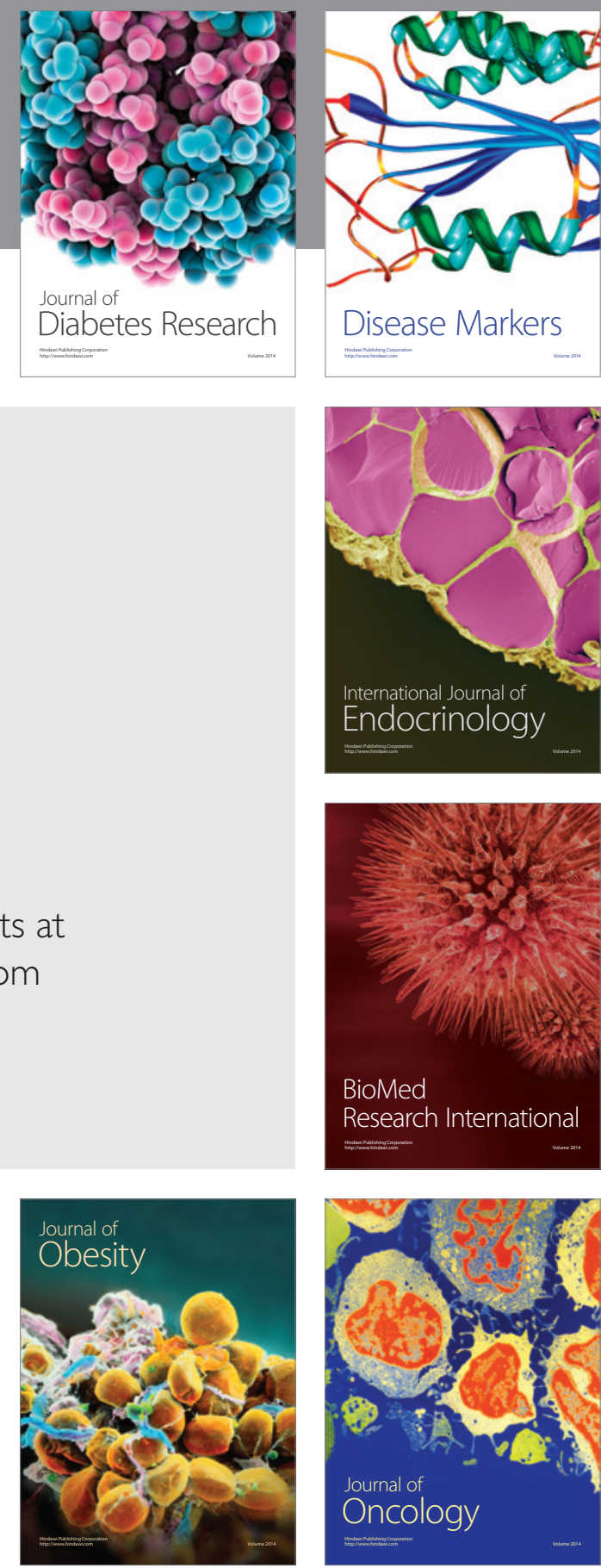

Disease Markers
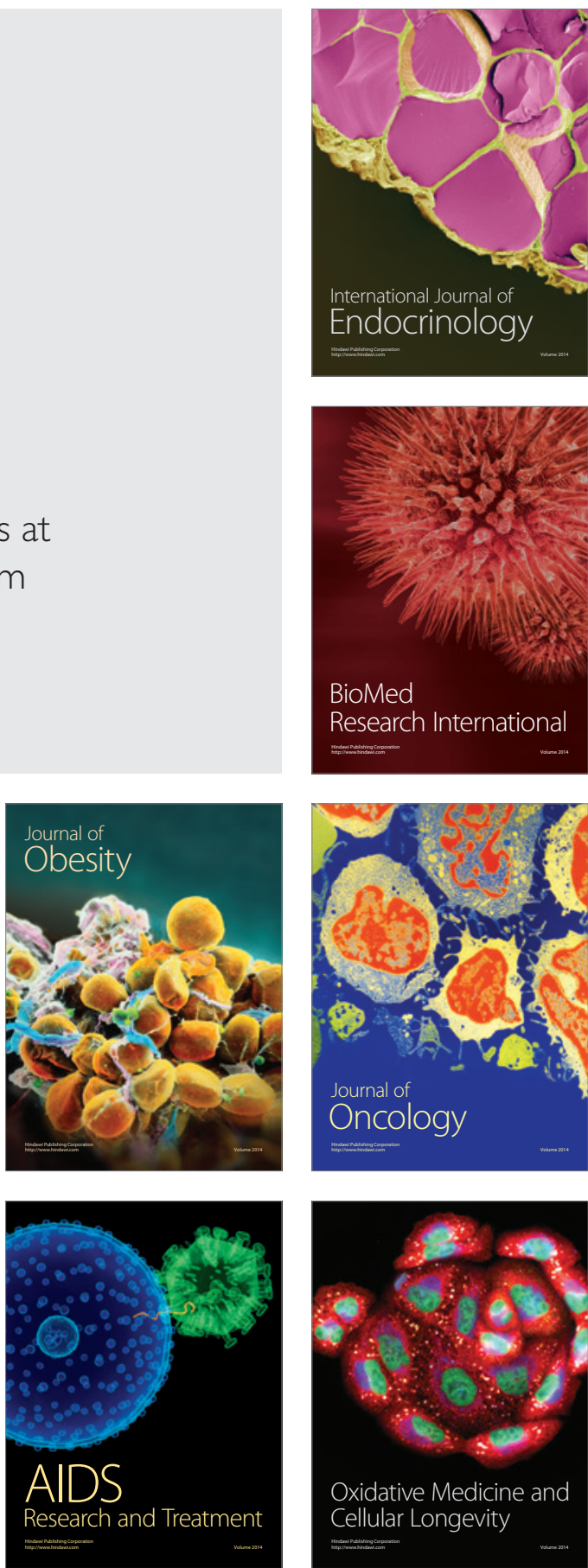